\title{
Is there a synergic effect of propafenone associated with atrial overdrive pacing for atrial arrhythmia prevention? A randomised crossover study
}

\author{
S Garrigue, S S Barold, S Cazeau, M Hocini, P Jais, M Haissaguerre, J Clementy
}

\begin{abstract}
Objective-To assess the effect of adding propafenone to atrial overdrive for the prevention of atrial arrhythmia episodes in patients with DDD pacemakers.

Design-22 patients ( 8 female, 14 male, mean (SD) age 67 (9) years, range 48 to 77) with DDD pacemakers and frequent paroxysmal atrial arrhythmia episodes were evaluated in a randomised crossover study.

Setting-University hospital.

Methods-Atrial overdrive was defined as a paced rate of 10 paced beats/min above the mean ventricular rate stored for the last 24 hours in the pacemaker memory function. The protocol consisted of two phases of one month each. The first phase consisted of atrial overdrive alone, while in the second phase, propafenone $(600 \mathrm{mg} / \mathrm{day})$ was added to atrial overdrive (atrial overdrive + propafenone). All 22 patients underwent the two phases in random order.

Results-Mean ventricular rate was $72(8)$ beats/min with atrial overdrive $v 73$ (6) with atrial overdrive + propafenone (NS). With atrial overdrive, 14 patients $(64.6 \%)$ had no recorded atrial arrhythmia $v 15(68.2 \%)$ with atrial overdrive + propafenone (NS). There was no statistical difference between the atrial overdrive and atrial overdrive + propafenone phases with regard to the number of atrial arrhythmia episodes (14 (27) v 13 (28)), their total duration (30 (78) v 29 (63) h), and their maximum duration (41 (72) $v 31$ (58) $\mathrm{min}$ ). However, in the brady-tachy subgroup with persistent atrial arrhythmias, atrial overdrive + propafenone produced a shorter mean cumulative duration of atrial arrhythmia than atrial overdrive (104 (115) $v 178$ (149) h, p = 0.04), with a significant decrease in the number of atrial arrhythmia episodes (134 (98) v102 (83), $\mathrm{p}=0.05)$. The proportion of asymptomatic atrial arrhythmia episodes increased only in the AV block group during atrial overdrive + propafenone $(\mathrm{p}=0.03)$. Three patients had atrial arrhythmias during atrial overdrive + propafenone but not with atrial overdrive alone.

Conclusions-In DDD paced patients, the overall effect of propafenone during atrial overdrive is variable. Propafenone may increase the proportion of asymptomatic atrial arrhythmia episodes. A proarrhythmic effect of propafenone was documented (aggravation of atrial arrhythmias). These results need to be confirmed by further larger randomised studies.

(Heart 2000;83:172-178)
\end{abstract}

Keywords: dual chamber pacing; atrial overdrive pacing; atrial arrhythmia; propafenone

Hôpital Cardiologique du Haut-Lévêque, University of Bordeaux, 19 avenue de Magellan, 33600 Pessac, France S Garrigue M Hocini P Jais M Haissaguerre J Clementy

University of Rochester School of Medicine and Dentistry, Rochester, New York, USA S S Barold

Centre Chirurgical du Val d'Or, Cardiac Pacing Department, Saint-Cloud, France S Cazeau

Correspondence to: Dr Garrigue email: stgarrigue@aol.com

Accepted 17 August 1999
Propafenone is a type IC antiarrhythmic drug commonly used for the treatment of atrial tachyarrhythmias. We recently documented the antiarrhythmic effect of atrial overdrive alone in the prevention of atrial arrhythmias during DDD pacing ${ }^{1}$ and postulated that the combination of atrial overdrive plus propafenone might be more effective than atrial overdrive alone. Consequently, we conducted a randomised prospective crossover study to evaluate the effect of propafenone in patients with DDD pacemakers undergoing atrial overdrive for atrial arrhythmia control.

\section{Methods}

PATIENTS

The study is an extension of our previously published protocol $^{1}$ for the evaluation of overdrive suppression of atrial arrhythmias by atrial pacing and involved the same 22 patients (eight women, 14 men, mean (SD) age, 67 (9) years, range 48 to 77 ) with DDD pacemakers (Chorus 6234, ELA Medical, Montrouge,
France) (table 1). Only the 11 patients with sick sinus syndrome $(n=5)$ and brady-tachy syndrome $(n=6)$ had received antiarrhythmic drugs before the study (range 5 to 13 months); five $(45 \%)$ were asymptomatic (absence of palpitations, dyspnoea, shortness of breath, or other symptoms consistent with arrhythmia) and the last 24 hour Holter recording just before the study did not reveal any ventricular or atrial arrhythmia. Four patients with bradytachy syndrome had been on amiodarone, and the washout was three months before inclusion in the study. The other seven patients on antiarrhythmic drugs underwent a washout period of at least five half lives. We then confirmed that all the patients had had atrial arrhythmias before atrial overdrive while not on antiarrhythmic drug treatment over a one month period. ${ }^{1}$ All were on warfarin. Atrial arrhythmia documentation, in terms of frequency and maximum and cumulative durations, was similar to our previous study. ${ }^{1}$ No patient developed chronic atrial fibrillation or 
Table 1 Clinical data

\begin{tabular}{|c|c|c|c|c|}
\hline Variables & $\begin{array}{l}\text { AV block } \\
(n=8) \uparrow\end{array}$ & $\begin{array}{l}S S \text { syndrome } \\
(n=5) \ddagger\end{array}$ & $\begin{array}{l}\text { BT syndrome } \\
(n=6) \rrbracket\end{array}$ & $\begin{array}{l}\text { CS syndrome } \\
(n=3)\end{array}$ \\
\hline Male sex & $5 \quad(62.5 \%)$ & $\begin{array}{ll}3 & (60.0 \%)\end{array}$ & $4 \quad(66.6 \%)$ & $(66.6 \%)$ \\
\hline Age (years) & $66.3(9.8)$ & $70.5(3.5)$ & $68.8(8.9)$ & $60.2(9.7)$ \\
\hline Hypertension $\dagger$ & $3 \quad(37.5 \%)$ & $2 \quad(40.0 \%)$ & $2 \quad(33.3 \%)$ & $1 \quad(33.3 \%)$ \\
\hline Coronary artery disease & $1 \quad(12.5 \%)$ & $0 \quad(0 \%)$ & $0 \quad(0 \%)$ & $0 \quad(0 \%)$ \\
\hline LVEF (\%) & $68 \quad(11)$ & $72 \quad(9)$ & $66 \quad(8)$ & $69 \quad(7)$ \\
\hline LA diameter (mm) & 33 (9) & $34 \quad(5)$ & $40 \quad(4)^{\star}$ & 32 (7) \\
\hline
\end{tabular}

This table is similar to that presented in the first part of the study ${ }^{1}$ and concerns the same 22 patients. Data are given as mean (SD) or $\mathrm{n}(\%)$. All antiarrhythmic drugs were discontinued in all 22 patients at the start of phase 1 of the previous protocol. ${ }^{1}$

${ }^{\star} \mathrm{p}<0.05$.

†Hypertension was well controlled in all patients.

$\ddagger$ Patients whose symptomatic atrial arrhythmias (AA) were controlled by antiarrhythmic drug treatment before entry into the phase 1 of the previous study. ${ }^{1}$

\Patients whose symptomatic atrial arrhythmic episodes were either partially controlled or not controlled by antiarrhythmic drug treatment before entry into the phase 1 of the previous study. IPatients with intermittent high degree AV block and long PR interval (>240 ms) the rest of the time.

$\mathrm{AV}$, atrioventricular; BT, brady-tachy (all patients with BT syndrome had documented SS syndrome); CS, carotid sinus; LA, left atrium; LVEF, left ventricular ejection fraction; SS, sick sinus.

atrial arrhythmias that persisted to the end of the one month period outlined below.

PROTOCOL

The first three phases represent the protocol of our previous study (fig 1). ${ }^{1}$ Phase IV is a

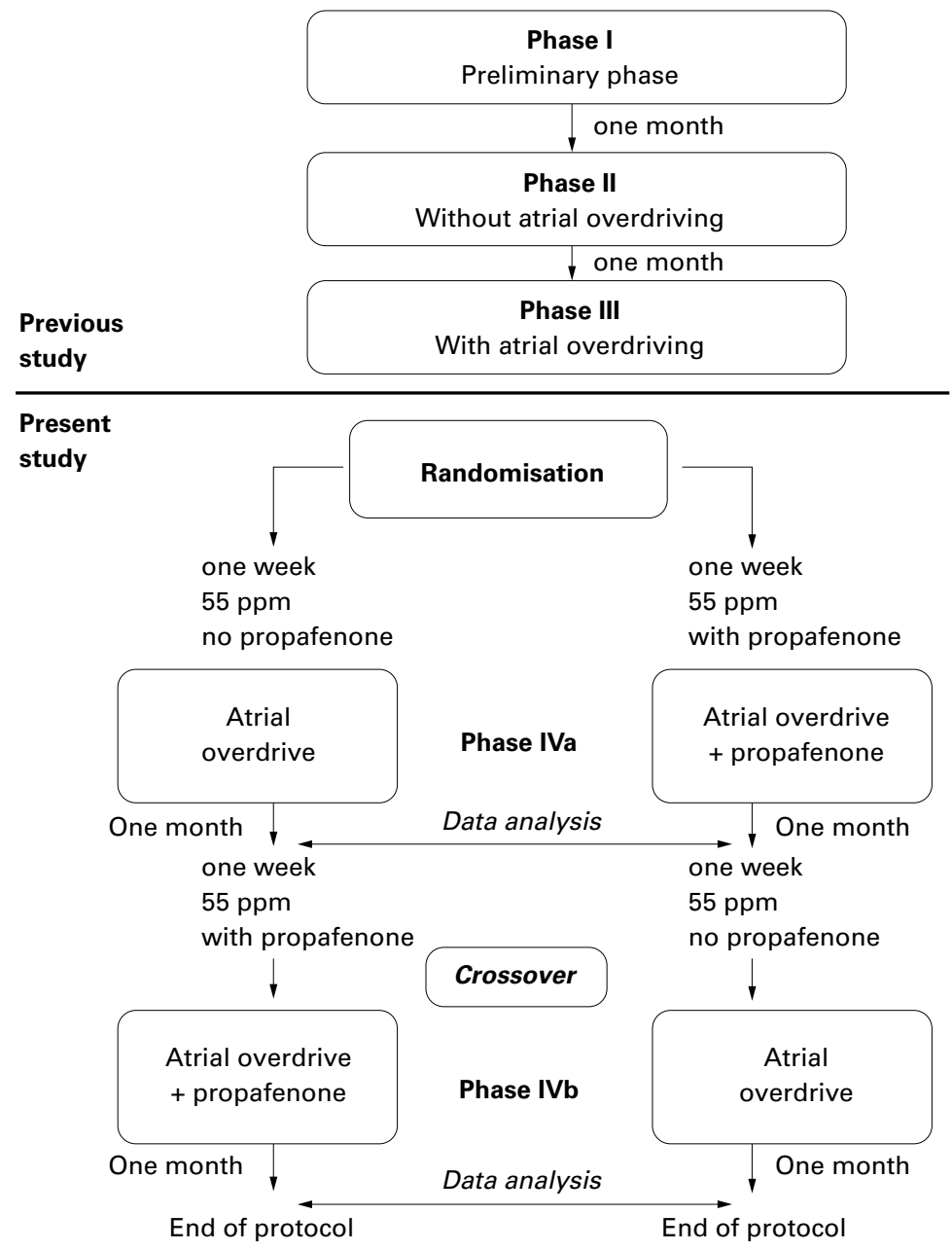

Figure 1 The previous study, depicted at the top, consisted of three phases of one month each. Clinical and pacemaker evaluations were performed at the beginning of each phase and at the end of phase III. The same 22 patients then underwent the present protocol consisting of a randomised crossover study with atrial overdrive alone versus atrial overdrive with propafenone during phase IVa and IVb. The pacemaker memory function was programmed for a one month period and results were assessed at the end of each phase. randomised crossover study to evaluate the effect of propafenone in patients with atrial arrhythmias treated by atrial overdrive. Atrial overdrive was defined as an atrial paced rate of 10 paced beats/min (ppm) above the mean ventricular rate stored for the previous 24 hours in the pacemaker memory. ${ }^{1}$

The patients were instructed not to change their daily life activities and to report palpitations, chest pain, dyspnoea, or other symptoms consistent with arrhythmias. A questionnaire was used at each visit to detect possible variations in patients' activities. Clinical and pacemaker evaluation were performed at the beginning of each phase. All symptoms consistent with arrhythmia were noted; the date of each symptomatic episode observed by the patient was analysed in terms of atrial arrhythmia identification in the pacemaker memory function. ${ }^{1}$ When symptoms occurred with an identified atrial arrhythmia, the episode was classified as a symptomatic atrial arrhythmia episode. When the pacemaker memory function identified an atrial arrhythmia without symptoms, the episode was classified as asymptomatic. Data collection and analysis were identical to those used in our previous study. ${ }^{1}$

\section{PROPAFENONE TREATMENT SCHEDULE}

After the conclusion of phase 3 of our previous study, all the DDD pacemakers were left programmed to the individual atrial overdrive rate and no antiarrhythmic agents were given. Fifteen days later, the pacemakers were reprogrammed to $55 \mathrm{ppm}$ and the device memory was activated. Twelve patients (group I) did not receive propafenone, while 10 patients (group II) received propafenone $(600 \mathrm{mg} / \mathrm{d}$ in divided doses). One week later ( $>5$ half lives of propafenone), the atrial overdrive rate was programmed (10 ppm above the memorised mean ventricular rate in the previous 24 hour period $^{1}$ ), thereby starting phase IVa, which lasted one month (fig 1). Upon completion of phase IVa, the pacemakers were reprogrammed to $55 \mathrm{ppm}$ and the device memory was activated. Propafenone was discontinued in group II patients and started $(600 \mathrm{mg} / \mathrm{d}$ in divided doses) in group I patients. After seven days, phase IVb, lasting one month, was begun. All the pacemakers were reprogrammed to the individual atrial overdrive rate as previously defined and the device memory was activated.

\section{PACEMAKER DATA}

At the beginning of phases IVa and IVb, when the overdrive rate was reprogrammed, the mean pacing rate varied slightly from patient to patient, depending on the phase (table 2). Nevertheless, the two mean overdrive rates were comparable and the differences were not significant. Other pacemaker data in phases IVa vs IVb were also not statistically different (table 2 ).

ATRIAL ARRHYTHMIA DETECTION

The diagnostic function of the Chorus 6234 pacemaker has been described in detail elsewhere. ${ }^{1}$ Briefly, this pacemaker senses spontaneous atrial cycles suddenly shortened by more than $25 \%$ from the preceding one, 
Table 2 Pacemaker data

\begin{tabular}{lll}
\hline & Phase IVa & Phase IVb \\
\hline Lower rate (ppm) & $72(8)$ & $73(6)$ \\
Upper rate (ppm) & $145(10)$ & $145(10)$ \\
AV delay (ms) (atrial sensing) & $141(13)$ & $141(13)$ \\
AV delay (ms) (atrial pacing) & $192(9)$ & $192(9)$ \\
PVARP (ms) & $234^{\star}$ & $234^{\star}$ \\
PV atrial blanking (ms) & $203^{\star}$ & $203^{\star}$ \\
Atrial sensitivity (mV) & $0.9(0.2)$ & $0.9(0.2)$ \\
Pulse duration (ms) & $0.49^{\star}$ & $0.49^{\star}$ \\
Bipolar atrial pacing threshold & & $1.3(0.3)$ \\
at the pulse width above (V) & $1.1(0.2)$ & 1.3 \\
\hline
\end{tabular}

*All 22 patients had the same value so the standard deviation equals 0 .

$\mathrm{AV}$, atrioventricular; ppm, paced beats/min; PV, postventricular; PVAR, postventricular atrial refractory period.

regardless of the prevailing heart rate or programmed parameters. Consequently, an atrial arrhythmia episode can be detected and registered at the time of onset, independently of the sinus rate. Two stages are involved for this sequence: first, a temporary diagnostic phase lasting 30 seconds during which the AV synchrony is limited to $120 \mathrm{ppm}$ or the programmed upper rate if slower; second, a fallback phase when the DDD mode is converted to the VVI (strictly the VDI) mode with a gradual reduction in the pacing rate to $70 \mathrm{ppm}$. The pacemaker memorises the total number of fallback episodes a month and the longest fallback duration. It also generates three separate histograms of all the interatrial, interventricular, and atrioventricular (AV) intervals for a total of 30 days. The histogram data allowed us to identify sustained atrial arrhythmia episodes and to determine the total duration of all arrhythmia episodes with a margin $<5 \%$.

DATA ACQUISITION

This study was considered as a paired statistical analysis for one group of patients (with atrial overdrive alone versus atrial overdrive + propafenone). Continuous variables are expressed as mean (SD) and compared by a paired non-parametric test (Wilcoxon paired W test). Qualitative variables are expressed as $\mathrm{n}$ (\%) and compared by the MacNemar's test. The coeffect of adding propafenone to the atrial overdrive was analysed by using a covariables model for each patient, using Epi-Info statistical software ${ }^{2}$

$\Delta=(\mathrm{a} \times \mathrm{A})+(\mathrm{b} \times \mathrm{B})$,

where $\Delta=$ difference between the number of atrial arrhythmia episodes with atrial overdrive and atrial overdrive associated with propafenone; $\mathrm{A}=$ atrial overdrive effect; $\mathrm{B}=$ atrial overdrive and propafenone effect; and a and $\mathrm{b}=$ coefficients quantifying the effects of $\mathrm{A}$ and $\mathrm{B}$, respectively.

A value of $p<0.05$ was considered significant.

\section{Results}

All patients tolerated the protocol. Neither ventricular tachyarrhythmias nor chronic atrial fibrillation were observed. The mean proportion of atrial pacing was 66 (3)\% in phase IV with atrial overdrive alone, compared with $66(11) \%$ in phase IV with atrial overdrive + propafenone (NS; table 3 ). The mean ventricular rate with atrial overdrive was 79 (7) beats/min $v 77$ (6) with atrial overdrive + propafenone (NS; table 3 ). If the patient subgroups are considered independently, the ventricular rate did not differ significantly between atrial overdrive and atrial overdrive + propafenone phases.

FREQUENCY OF ATRIAL ARRHYTHMIAS

In the 22 patients taken as a group, atrial overdrive was associated with a total of 14 (27) atrial arrhythmia episodes, $v 13$ (28) with atrial overdrive + propafenone (NS; table 3). During atrial overdrive, eight of 22 patients $(36.4 \%$; four with AV block and four with the brady-tachy syndrome) had atrial arrhythmias, while during atrial overdrive + propafenone, seven of 22 patients $(31.8 \%$; three with AV block, three with the brady-tachy syndrome, and one with sick sinus syndrome) had atrial arrhythmias (NS) (fig 2).

In the 15 patients (seven with atrial overdrive + propafenone, eight with atrial overdrive alone) who presented with persistent atrial arrhythmias, we observed a significant expo-

Table 3 Atrial arrhythmia episodes

\begin{tabular}{|c|c|c|c|c|c|c|}
\hline \multirow{2}{*}{ Atrial overdrive, phase IVa or IVb } & \multirow{2}{*}{$\begin{array}{l}\text { AV block } \\
(n=8)\end{array}$} & \multicolumn{2}{|c|}{$\begin{array}{l}\text { SS syndrome } \\
(n=5)\end{array}$} & \multirow[t]{2}{*}{$\begin{array}{l}\text { BT syndrome } \\
(n=6)\end{array}$} & \multirow[t]{2}{*}{$\begin{array}{l}\text { CS syndrome } \\
(n=3)\end{array}$} & \multirow[t]{2}{*}{$\begin{array}{l}\text { Total } \\
(n=22)\end{array}$} \\
\hline & & & & & & \\
\hline Heart rate $($ beats $/ \mathrm{min}) \ddagger$ & $84(5)$ & 68 & (6) & $76(5)$ & $83(7)$ & $79(7)$ \\
\hline Patients without AA & $4(50.0 \%)$ & 5 & $(100 \%)$ & $2(33.3 \%)$ & $3(100 \%)$ & $14(63.6 \%)$ \\
\hline Patients with AA & $4(50.0 \%)$ & 0 & & $4(66.6 \%)$ & 0 & $8(36.4 \%)$ \\
\hline Number of AA episodes $\dagger$ & $5(2)$ & 0 & & $68(97)$ & 0 & $14(27)$ \\
\hline Proportion of atrial pacing $(\%) \mathbb{S}$ & $66(4)$ & 71 & (8) & $63(9)$ & $65(3)$ & $66(3)$ \\
\hline Total duration of AA episodes (hours) & $4(2)$ & - & & $104(126)$ & - & $30(78)$ \\
\hline During of longest AA episode (min) & $81(71)$ & - & & $112(81)$ & - & $41(72)$ \\
\hline \multicolumn{7}{|c|}{ Atrial overdrive and propafenone, phase IVa or IVb } \\
\hline Heart rate (beats $/ \mathrm{min}) \ddagger$ & $78(5)^{\star}$ & 74 & $(5)^{\star}$ & $75(6)$ & $82(6)$ & $77(6)$ \\
\hline Patients without AA & $5(62.5 \%)$ & 4 & $(80 \%)$ & $3(50 \%)$ & $3(100 \%)$ & $15(68.2 \%)$ \\
\hline Patients with AA & $3(37.5 \%)$ & 1 & $(20 \%)$ & $3(50 \%)$ & 0 & $7(31.8 \%)$ \\
\hline Number of AA episodes $\dagger$ & $3(1)$ & 1 & & $68(53)$ & 0 & $13(28)$ \\
\hline Proportion of of atrial pacing $(\%) \rrbracket$ & $62(12)$ & 78 & (12) & $66(7)$ & $63(7)$ & $66(11)$ \\
\hline Total duration of AA episodes (h) & $2(3)$ & 0.2 & & $57(85)^{\star}$ & - & $29(63)$ \\
\hline During of longest AA episode (min) & $69(72)$ & 28 & & $91(102)$ & - & $31(58)$ \\
\hline
\end{tabular}

Data are mean (SD) or percentage.

${ }^{\star} \mathrm{p}<0.05$ between patients with atrial overdrive and patients with atrial overdrive and propafenone.

†Number determined by the fallback count in the memory of the pacemaker.

¥Ventricular.

$§$ Percentage of all atrial beats.

AA, atrial arrhythmia; AV, atrioventricular; BT, brady-tachy; CS, carotid sinus; SS, sick sinus. 


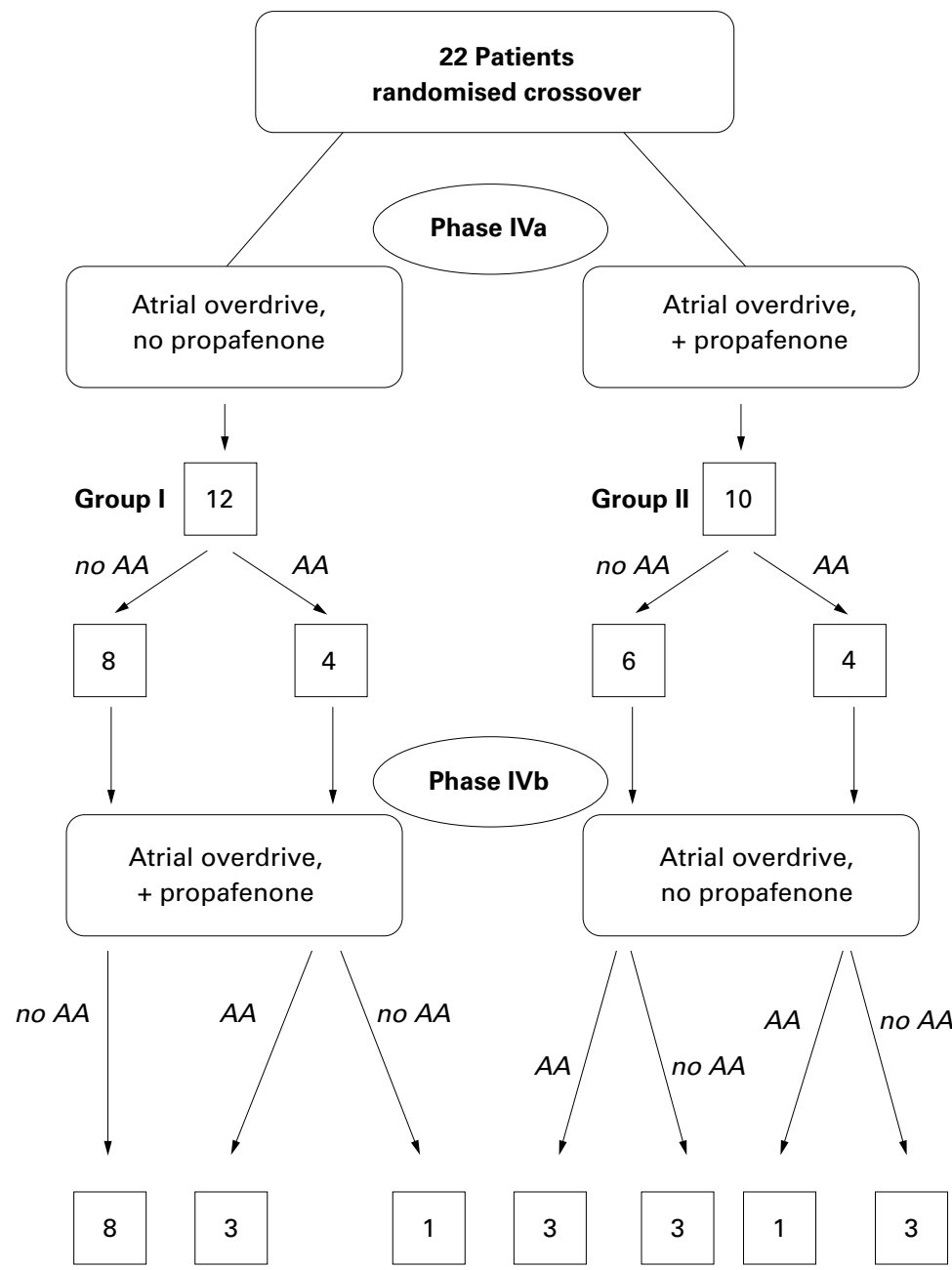

Figure 2 Overall results. During phase IVa, 12 patients (group I) were randomised to atrial overdrive alone while 10 (group II) underwent atrial overdrive with propafenone. At the end of the crossover, four patients had atrial arrhythmia episodes in phases IVa and $I V b$, and 11 patients had no atrial arrhythmia episode in either phase. Three patients had atrial arrhythmias with atrial overdrive + propafenone but not with atrial overdrive alone. Four patients had atrial arrhythmia episodes during atrial overdrive but not during atrial overdrive with propafenone. AA, atrial arrhythmias.

nential correlation between the number of atrial arrhythmia episodes and the percentage of atrial pacing $(r=0.95 ; \mathrm{p}<0.001)$. The mean percentage of atrial pacing in patients with atrial arrhythmia episodes during atrial overdrive and/or atrial overdrive + propafenone was statistically similar to that of patients with- out atrial arrhythmia episodes (65 (12)\% v 56 (21)\%, respectively; NS), showing that the presence of atrial arrhythmia episodes did not influence the percentage of atrial pacing from patient to patient.

CUMULATIVE DURATION OF ATRIAL ARRHYTHMIA EPISODES

Analysis of patient subgroups showed a similar mean cumulative duration of atrial arrhythmias in all eight patients in the AV block group (4 (2) hours in phase IV with atrial overdrive $v 2$ (3) hours in phase IV with atrial overdrive + propafenone, NS). In contrast, the group of six patients with brady-tachy syndrome had a significantly shorter mean cumulative duration of atrial arrhythmias during atrial overdrive + propafenone than during atrial overdrive alone (57 (85) hours with atrial overdrive + propafenone $v 104$ (126) hours with atrial overdrive; $\mathrm{p}=0.02$ ) (table 3$)$. No patient with sick sinus syndrome or carotid sinus syndrome developed atrial arrhythmias with atrial overdrive, while one patient with sick sinus syndrome had one short atrial arrhythmia episode lasting $0.2 \mathrm{~h}$ during atrial overdrive + propafenone (table 3 ).

\section{MAXIMUM DURATION OF ATRIAL ARRHYTHMIA} EPISODES

For all the 22 patients, the maximum duration of atrial arrhythmia episodes was statistically similar in both phases (41 (72) $\mathrm{min}$ in phase IV with atrial overdrive $v 31$ (58) $\mathrm{min}$ in phase IV with atrial overdrive + propafenone) (table 3 ). In the patient subgroups there was also no significant difference in the longest atrial arrhythmia episode duration for patients with AV block (81 (71) min in phase IV with atrial overdrive $v 69$ (72) min in phase IV with atrial overdrive + propafenone), or for patients with brady-tachy syndrome (112 (81) $\mathrm{min}$ in phase IV with atrial overdrive $v 91$ (102) $\mathrm{min}$ in phase IV with atrial overdrive + propafenone) (table 3).

\section{SYMPTOMS}

No patient complained of dizziness or syncope during the protocol (table 4). In eight patients propafenone was associated with minor side effects not requiring discontinuation (bitter taste in five, nausea in two, and weakness

Table 4 Symptoms during atrial arrhythmia episodes

\begin{tabular}{|c|c|c|c|c|c|}
\hline & $\begin{array}{l}\text { AV block } \\
(n=8)\end{array}$ & $\begin{array}{l}\text { SS syndrome } \\
(n=5)\end{array}$ & $\begin{array}{l}\text { BT syndrome } \\
(n=6)\end{array}$ & $\begin{array}{l}\text { CS syndrome } \\
(n=3)\end{array}$ & $\begin{array}{l}\text { Total } \\
(n=22)\end{array}$ \\
\hline \multicolumn{6}{|l|}{ Atrial overdrive, phase IVa or IVb } \\
\hline Patients with AA & $4(50 \%)$ & 0 & $4(66 \%)$ & 0 & $8(36.4 \%)$ \\
\hline Patients with symptoms & $4(100 \%)$ & & $4(100 \%)$ & & $8(36.4 \%)$ \\
\hline \multirow[t]{2}{*}{ Number of symptomatic events } & $1(1)(\mathrm{D})$ & & 9 (7) (D) & & 7 (5) (D) \\
\hline & $3(1)(P)$ & & $13(0)(P)$ & & $11(6)(P)$ \\
\hline Number of asymptomatic AA episodes & $0.5(1.5) ; 23 \%$ & & $47(21) ; 69 \%$ & & $16(10) ; 46 \%$ \\
\hline \multicolumn{6}{|c|}{ Atrial overdrive and propafenone, phase IVa or IVb } \\
\hline Patients with AA & $3(37.5 \%)$ & $1(20 \%)$ & $3(50.0 \%)$ & 0 & $7(31.8 \%)$ \\
\hline Patients with symptoms & $3(100 \%)$ & $1(100 \%)$ & $3(100 \%)$ & $1 \dagger$ & $8(36.4 \%)$ \\
\hline \multirow[t]{3}{*}{ Number of symptomatic events } & $1(2)(\mathrm{D})$ & 1 (D) & 1 (DT) & $2(\mathrm{D}) \dagger$ & 1 (DT) \\
\hline & & & 9 (8) (D) & & 5 (6) (D) \\
\hline & & & $8(11)(P)$ & & $3(5)(P)^{\star}$ \\
\hline Number of asymptomatic AA episodes & 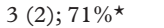 & 0 & $49(18) ; 72 \%$ & 0 & $15(8) ; 58 \%$ * \\
\hline
\end{tabular}

Data are mean (SD) or percentage.

${ }^{\star} \mathrm{p}<0.05$ between patients with atrial overdrive and patients with atrial overdrive and propafenone.

+Patient with no atrial arrhythmic episode recording in the memory function of the PM.

AA, atrial arrhythmia; AV, atrioventricular; BT, brady-tachy; CP, chest pain; CS, carotid sinus; D, dyspnoea; P, palpitations; SS, sick sinus. 
in one). Analysis of the 22 patients showed that the frequency of asymptomatic atrial arrhythmia episodes was greater with atrial overdrive + propafenone than with atrial overdrive alone $(58 \%$ v $46 \%, \mathrm{p}=0.048)$. The brady-tachy syndrome group showed the highest percentage of asymptomatic atrial arrhythmia episodes in phase IV with atrial overdrive and in phase IV with atrial overdrive + propafenone $(69 \%$ v $72 \%$, respectively), but the difference was not statistically significant (table 4). In contrast, patients with AV block had a statistically significant increase in asymptomatic episodes with atrial overdrive + propafenone $v$ atrial overdrive $(71 \%$ v 23\%, $\mathrm{p}=0.02)($ table 4$)$.

EFFECT OF PROPAFENONE WITH ATRIAL

OVERDRIVE

A potential synergic effect of propafenone with atrial overdrive was evaluated. Covariable analysis between atrial overdrive alone and atrial overdrive + propafenone showed no specific propafenone effect in reducing the number of atrial arrhythmia episodes in the whole population $(n=22)$. Fourteen patients had no atrial arrhythmia episodes with atrial overdrive $v 15$ patients with atrial overdrive + propafenone (NS). Only four patients had atrial arrhythmia episodes during both atrial overdrive and atrial overdrive + propafenone (fig 2 ).

EFFECT OF PROPAFENONE IN PATIENTS WITH PERSISTENT ATRIAL ARRHYTHMIA DURING ATRIAL OVERDRIVE

During atrial overdrive + propafenone, three patients with brady-tachy syndrome had persistent atrial arrhythmia episodes $v$ four during

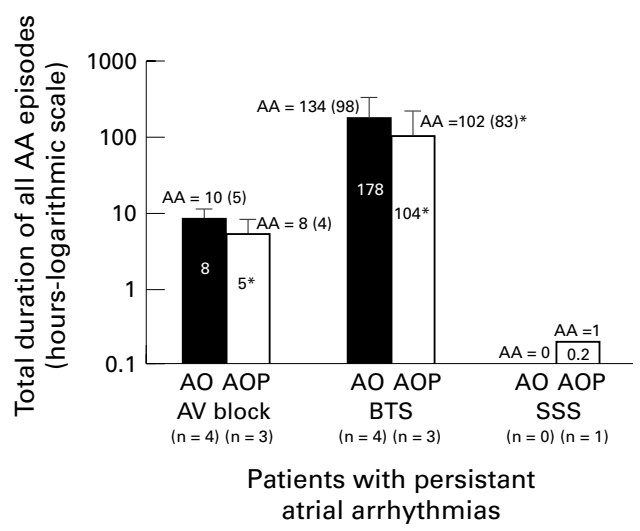

Figure 3 Mean cumulative duration of atrial arrhythmias in the seven patients with atrial arrhythmia episodes during atrial overdrive + propafenone (three with atrioventricular $(A V)$ block, three with brady-tachy syndrome (BTS), and one with sick sinus syndrome (SSS)) (white columns) compared with the eight patients with atrial arrhythmia episodes during atrial overdrive alone (four with AV block, four with BTS) (black columns). Error bars $=$ SEM. The respective mean cumulative duration of all atrial arrhythmia episodes (hours) is given inside each bar. During atrial overdrive, propafenone significantly decreased the cumulative duration of atrial arrhythmia episodes in patients with AV block and BTS, as well as the mean number of atrial arrhythmia episodes in patients with BTS (shown above the bars). Note that with propafenone, one patient with SSS had a short atrial arrhythmia episode (12 minutes), while no atrial arrhythmia was identified with atrial overdrive alone. AA, mean number of atrial arrhythmia episodes (SD); ${ }^{\star} p<0.05$ between phases with atrial overdrive $v$ atrial overdrive + propafenone. atrial overdrive alone (NS; table 3). However, in this specific brady-tachy subgroup with persistent atrial arrhythmias, the atrial overdrive + propafenone phase showed a significantly shorter cumulative duration of all atrial arrhythmia episodes than the phase with atrial overdrive alone (104 (115) v 178 (149) hours, respectively; $p=0.04$ ) (fig 3 ). Moreover, in the same group, the mean number of atrial arrhythmias was significantly smaller during the atrial overdrive + propafenone phase compared with atrial overdrive alone (102 (83) $v$ 134 (98), $\mathrm{p}=0.05$ ) (fig 3).

In the AV block subgroup with persistent atrial arrhythmias (fig 3), the atrial overdrive + propafenone phase exhibited a significant reduction in terms of atrial arrhythmias cumulative duration (5 (3) $v 8$ (2) hours, $\mathrm{p}=0.04$ ) but without significant difference in the mean number of atrial arrhythmia episodes (8 (4) during atrial overdrive $v 10$ (5) during atrial overdrive + propafenone, NS).

EFFECT OF PROPAFENONE IN PATIENTS WITHOUT ATRIAL ARRHYTHMIAS DURING ATRIAL OVERDRIVE Three patients had no atrial arrhythmias during phase IV with atrial overdrive, while atrial arrhythmia episodes were observed during phase IV with atrial overdrive + propafenone. In these patients, one (AV block) developed three short atrial arrhythmia episodes (the longest being 32 minutes), only one of which was asymptomatic. Another patient (with brady-tachy syndrome) had eight atrial arrhythmia episodes with a cumulative duration of 16 hours: four were asymptomatic. The last patient (sick sinus syndrome) had one short symptomatic atrial arrhythmia episode of 28 minutes. All three had a comparable percentage of atrial pacing in phases IVa and IVb (respectively $66 \%$ v $64 \%$ for the patient with AV block, $72 \%$ v $69 \%$ for the patient with brady-tachy syndrome patient, and 74\% v 76\% for the patient with sick sinus syndrome).

\section{Discussion}

In our previous study, with a modest degree of atrial overdrive and no antiarrhythmic agents, atrial arrhythmias were prevented in 14 of our 22 patients and atrial arrhythmias were reduced in frequency and cumulative duration in the other eight patients (four with brady-tachy syndrome and four with AV block). ${ }^{1}$ This was the starting point of the present study in which the combination of atrial overdrive and propafenone produced a total of 15 patients without atrial arrhythmias. In the entire cohort of 22 patients, there was no statistically significant reduction in the number of atrial arrhythmias and in the longest and cumulative durations of atrial arrhythmia episodes with propafenone. However, in the brady-tachy group with persistent atrial arrhythmias despite atrial overdrive, propafenone caused a significant reduction in the number of atrial arrhythmia episodes (134 (98) v 102 (83), $\mathrm{p}=0.05$ ) and in the cumulative atrial arrhythmia duration from 178 (149) to 104 (115) hours ( $\mathrm{p}=0.04)$. Likewise, in the AV block subgroup with persistent atrial arrhythmias despite atrial overdrive, 
propafenone reduced the cumulative atrial arrhythmia duration significantly (5 (3) $v 8$ (2) hours; $\mathrm{p}=0.04)$.

ASYMPTOMATIC ATRIAL ARRHYTHMIA EPISODES When compared with the atrial overdrive phase, the atrial overdrive + propafenone phase showed a significant increase in the number of asymptomatic atrial arrhythmia episodes in the overall cohort, but mainly in patients with $\mathrm{AV}$ block $(23 \%$ v $71 \% ; \mathrm{p}=0.03)$ and not in the brady-tachy patient subgroup. This increase of asymptomatic atrial arrhythmias in the AV block group probably reflected a decrease in ventricular rate, as suggested by Pritchett et al. ${ }^{3}$ This suggests that if only symptomatic atrial arrhythmia episodes are identified, the true number of atrial arrhythmia episodes will be underestimated in patients treated with propafenone or other antiarrhythmic agents.

EFFECT OF PROPAFENONE

In this study, a fixed dose of propafenone (600 $\mathrm{mg} /$ day) was used without titration. Recently, Pritchett et al have shown in a large population that there was no significant difference between a propafenone dose of $900 \mathrm{mg} /$ day and 600 $\mathrm{mg} /$ day in terms of plasma concentration over a one month period. ${ }^{3}$

Three patients with atrial arrhythmias in the atrial overdrive + propafenone phase eventually became free of atrial arrhythmias during the atrial overdrive phase. We cannot explain why this paradoxical effect was not observed when the sequence was reversed, starting with the atrial overdrive phase in atrial arrhythmiafree patients. The difference may reflect the small number of patients in the study. Nevertheless, the worsening of atrial arrhythmia episodes appears to represent a proarrhythmic effect of propafenone, previously reported on a much smaller scale in patients treated for atrial arrhythmias without a pacemaker. ${ }^{4-6}$ Earlier studies probably underestimated the atrial proarrhythmic effect of propafenone because of their limited monitoring techniques (24 hour Holter surface ECG, compared with the long term pacemaker memory data in our study).

LIMITATIONS OF THE STUDY

This was a pilot study with a small number of patients. We are conscious of the weak statistical power in the study. However, our population of 22 patients had sufficient power to detect a difference of $25 \%$ or more between the proportion of patients free of atrial arrhythmias with atrial overdrive alone, versus those with atrial overdrive + propafenone. ${ }^{78}$ The level of significance $(\mathrm{p}<0.05)$ was provided at a statistical power $\left(1-\beta\right.$ risk) of $60 \% .{ }^{78}$ Patients were prospectively randomised so that, independently of time, three cases of potential proarrhythmic effects of propafenone were observed, while with atrial overdrive + propafenone versus atrial overdrive alone a difference of only one patient free of atrial arrhythmias was shown. Our observations in a relatively small population obviously need to be confirmed in larger randomised studies.

\section{CONCLUSIONS}

Our study has shown the variable effect of propafenone in patients with DDD pacemakers treated with atrial overdrive for atrial arrhythmia prevention. Overall the antiarrhythmic effect of propafenone was disappointing, though it may be useful in increasing the proportion of asymptomatic atrial arrhythmias in symptomatic patients. Such results might lead to a re-evaluation of the antiarrhythmic agent efficacy in terms of atrial arrhythmia suppression. In view of the potential proarrhythmic effects of propafenone, patients with atrial arrhythmias refractory to propafenone should be treated with atrial overdrive only after propafenone has been discontinued. Similarly, patients with atrial arrhythmias refractory to both atrial overdrive and propafenone should be re-evaluated by atrial overdrive alone to rule out a possible propafenone proarrhythmic effect.

1 Garrigue S, Barold SS, Cazeau S, et al. Prevention of atrial arrhythmias during DDD pacing by atrial overdrive. $P A C E$ 1998;211751-9.

2 Dean $\mathrm{AD}$, Dean JA, Burton JH, et al. Epi Info, version 5: a word processing, database, and statistics program for epidemiology on micro-computers. Atlanta, Georgia: Centers for ogy on micro-computers. Atlan
Disease Control, 1990:82-5.

3 Pritchett ELC, McCarthy EA, Wilkinson WE. Propafenone treatment of symptomatic paroxysmal supraventricular arrhythmias: a randomized, placebo-controlled, crossover arrhythmias: a randomized, placebo-controlled, crossover trial in patients to

4 Kyles AE, Murdock CJ, Yeung-Lai-Wah JA, et al. Long-term efficacy of propafenone for prevention of atrial fibrillation. Can $\mathcal{F}$ Cardiol 1991;7:407-9.

5 Hammill SC, Wood DL, Gersh BJ, et al. Propafenone for paroxysmal atrial fibrillation. Am f Cardiol 1988;61:473-4. 6 Podrid PJ, Anderson JL, for the Propafenone Multicenter Study Group. Safety and tolerability of long-term propafenone therapy for supraventricular tachyarrhythmias. Am $\mathcal{F}$ Cardiol 1996;78:430-4.

7 Schlesselman JJ. Sample size requirements in cohort and case-control studies of disease. Am f Epidemiol 1974;99: $381-4$.

8 Rothman KJ, Boice JD. Epidemiologic analysis with a programmable calculator, 2 nd ed. Chestnut Hill, MA: Epidemiology Resources Inc, 1982 . 